\title{
Reductive and Catalytic Monoalkylation of Primary Amines Using Nitriles as an Alkylating Reagent
}

\author{
Hironao Sajiki,* Takashi Ikawa, Kosaku Hirota* \\ Laboratory of Medicinal Chemistry, Gifu Pharmaceutical University, Gifu 502-8585, \\ Japan
}

Supporting Information

Table of Contents:

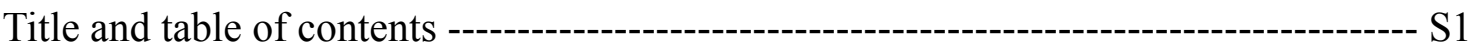

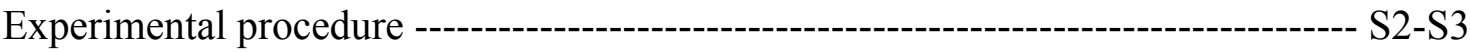

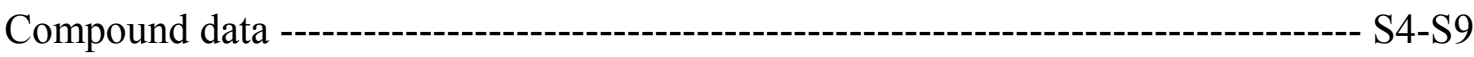

References --------------------------------------------------------------------------------------- S10

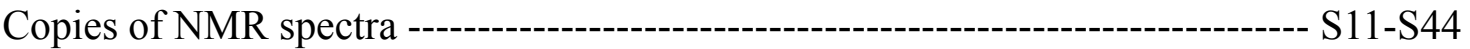


Purification method of nitriles. ${ }^{1}$ Each nitrile purchased from Aldrich, Nacalai, TCI and Wako (98-99.8\% purity) was washed with conc $\mathrm{HCl}$ and brine, then dried over $\mathrm{K}_{2} \mathrm{CO}_{3}$. The organic layer was fractionally distilled from $\mathrm{P}_{2} \mathrm{O}_{5}$.

General procedure for reductive alkylation of aromatic amines. After two vacuume/ $\mathrm{H}_{2}$ cycles to remove air from the reaction tube, the stirred mixture of the aromatic amine $(1.0$ or $0.5 \mathrm{mmol}), 10 \% \mathrm{Pd} / \mathrm{C}(10 \mathrm{wt} \%$ of the amine) and the nitrile $(5$ equiv) in $\mathrm{MeOH}(1 \mathrm{~mL})$ was hydrogenated at ordinary pressure (balloon) and temperature (ca. $20^{\circ} \mathrm{C}$ ) for appropriate time (see Table 1, 2 and 3). The reaction mixture was filtrated using a membrane filter (Millipore, Millex ${ }^{\circledR}-\mathrm{LH}, 0.45 \mu \mathrm{m}$ ) and the filtrate was concentrated under reduced pressure. The ratio of the primary (1), secondary (2) and tertiary amines (3) was confirmed by ${ }^{1} \mathrm{H}$ NMR of the crude mixture in $\mathrm{CDCl}_{3}$. The crude mixture was purified by flash silica gel column chromatography, if necessary.

When $\mathrm{NH}_{4} \mathrm{OAc}$ was used as an additive, the residue was partitioned between ether (10 $\mathrm{mL})$ and water $(10 \mathrm{~mL})$. The aqueous layer was extracted with ether $(10 \mathrm{~mL} \times 3)$, then combined organic layers were washed with brine $(10 \mathrm{~mL})$, dried with anhydrous $\mathrm{Na}_{2} \mathrm{SO}_{4}$, filtered and concentrated under reduced pressure.

Procedure for reductive cyclization of 2-aminobenzylnitrile (7) (equation 1) After two vacume $/ \mathrm{H}_{2}$ cycles to remove air from the reaction tube, the stirred mixture of the 2-aminobenzylcyanide (7) $(81.1 \mathrm{mg}, 0.5 \mathrm{mmol}), 10 \% \mathrm{Pd} / \mathrm{C}(8.1 \mathrm{mg}, 10 \mathrm{wt} \%$ of the substrate) in $\mathrm{MeOH}(1 \mathrm{~mL})$ was hydrogenated at ordinary pressure (balloon) and temperature (ca. $20^{\circ} \mathrm{C}$ ) for $22 \mathrm{~h}$. The reaction mixture was filtrated using a membrane filter (Millipore, Millex ${ }^{\circledR}-\mathrm{LH}, 0.45 \mu \mathrm{m}$ ) and filtrate was concentrated under reduced pressure. The residue was purified by column chromatography on silica gel (hexane/ether 20:1) to afford indole (8) (62.4 $\mathrm{mg}, 98 \%$ yield) as a white solid.

\section{General procedure for preparation of mono-alkylaniline derivatives from} nitrobenzene (9). After two vacuume/ $\mathrm{H}_{2}$ cycles to remove air from the reaction tube, the stirred mixture of the nitrobenzene (9) $(61.6 \mathrm{mg}, 0.5 \mathrm{mmol}), 10 \% \mathrm{Pd} / \mathrm{C}(6.2 \mathrm{mg}, 10$ $\mathrm{wt} \%$ of the amine) and nitrile (5 equiv) in $\mathrm{MeOH}(1 \mathrm{~mL})$ was hydrogenated at ordinary pressure (balloon) and at temperature (ca. $20^{\circ} \mathrm{C}$ ) for the appropriate time (see Table 4). The reaction mixture was filtrated using a membrane filter (Millipore, Millex ${ }^{\circledR}$-LH, 0.45 $\mu \mathrm{m})$ and filtrate was concentrated under reduced pressure. The ratio of the primary (1), secondary (2) and tertiary amines (3) was confirmed by ${ }^{1} \mathrm{H}$ NMR of the crude mixture in $\mathrm{CDCl}_{3}$. The crude mixture was purified by flash silica gel column chromatography, if 
necessary.

When $\mathrm{NH}_{4} \mathrm{OAc}$ was used as an additive, the residue was partitioned between ether (10 $\mathrm{mL})$ and water $(10 \mathrm{~mL})$. The aqueous layer was extracted with ether $(10 \mathrm{~mL} \times 3)$, then combined organic layers were washed with brine $(10 \mathrm{~mL})$, dried with anhydrous $\mathrm{Na}_{2} \mathrm{SO}_{4}$, filtered and concentrated under reduced pressure.

General procedure for reductive alkylation of aliphatic amines. After two vacuume $/ \mathrm{H}_{2}$ cycles to remove air from the reaction tube, the stirred mixture of the aliphatic amine $(1.0 \mathrm{mmol}), 10 \% \mathrm{Pd} / \mathrm{C}$ or $5 \% \mathrm{Rh} / \mathrm{C}(10 \mathrm{wt} \%$ of the amine $)$ and a nitrile ( 5 equiv or 2 equiv) in $\mathrm{MeOH}(1 \mathrm{~mL})$ was hydrogenated at ordinary pressure (balloon) and temperature (ca. $20^{\circ} \mathrm{C}$ ) for the appropriate time (see Table 5). The reaction mixture was filtrated using a membrane filter (Millipore, Millex ${ }^{\circledR}-\mathrm{LH}, 0.45 \mu \mathrm{m}$ ) and filtrate was concentrated under reduced pressure. The ratio of the primary (10), secondary (11) and tertiary amines (12) was confirmed by ${ }^{1} \mathrm{H}$ NMR of the crude mixture in $\mathrm{CDCl}_{3}$. The crude mixture was purified by flash silica gel column chromatography, if necessary.

When $\mathrm{NH}_{4} \mathrm{OAc}$ was used as an additive, the residue was partitioned between ethylacetate $(10 \mathrm{~mL})$ and water $(10 \mathrm{~mL})$. The aqueous layer was extracted with ether $(10 \mathrm{~mL} \times 3)$, then combined organic layers were washed with brine $(10 \mathrm{~mL})$, dried with anhydrous $\mathrm{Na}_{2} \mathrm{SO}_{4}$, filtered and concentrated under reduced pressure.

Procedure for synthesis of $N$-ethyl- $N$-propyl-2-phenylethylamine (15). After two vacuume $/ \mathrm{H}_{2}$ cycles to remove air from the reaction tube, the stirred mixture of the 2-phenylethylamine (13) (121.2 mg, $1.0 \mathrm{mmol}), 5 \% \mathrm{Rh} / \mathrm{C}(24.2 \mathrm{mg}, 20 \mathrm{wt} \%$ of the amine) and acetonitrile $(261 \mu \mathrm{l}, 5.0 \mathrm{mmol})$ in $\mathrm{MeOH}(1 \mathrm{~mL})$ was hydrogenated under ambient pressure (balloon) and at room temperature $\left(\right.$ ca. $20^{\circ} \mathrm{C}$ ) for $28 \mathrm{~h}$. The reaction mixture was filtrated using a membrane filter (Millipore, Millex ${ }^{\circledR}-\mathrm{LH}, 0.45 \mu \mathrm{m}$ ) and filtrate was concentrated under reduced pressure. The reaction mixture and distilled propionitrile $(357 \mu \mathrm{l}, 5.0 \mathrm{mmol})$ in $\mathrm{MeOH}$ was hydrogenated in the presence of $10 \%$ $\mathrm{Pd} / \mathrm{C}(16.3 \mathrm{mg})$ and ammonium acetate $(77.1 \mathrm{mg}, 1.0 \mathrm{mmol})$ under ambient temperature and pressure for $7 \mathrm{~h}$. The reaction mixture was filtrated using a membrane filter (Millipore, Millex ${ }^{\circledR}$-LH, $0.45 \mu \mathrm{m}$ ) and filtrate was concentrated under reduced pressure. The residue was partitioned between ethyl acetate $(10 \mathrm{~mL})$ and water $(10 \mathrm{~mL})$. The aqueous phase was extracted with ethyl acetate $(10 \mathrm{~mL} \times 3)$, then combined the organic phases were washed with brine $(10 \mathrm{~mL})$, dried with anhydrous $\mathrm{Na}_{2} \mathrm{SO}_{4}$, filtered and concentrated under reduced pressure to afford $N$-ethyl- $N$-propyl-2-phenylethylamine ${ }^{2}$ (89.5 mg, 94\%). 
NHEt

$\boldsymbol{N}$-Ethylaniline (Table 1, entry 3 and Table 2, entry 1, commercially available)<smiles>CCCNc1ccccc1</smiles>

$\boldsymbol{N}$-Propylaniline (Table 2 , entry 2 , commercially available)

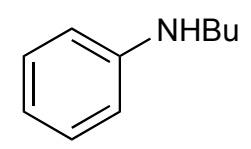

$\boldsymbol{N}$-Butylaniline (Table 2 , entry 4 and Table 4 , entry 1 , commercially available)<smiles>CCCCCNc1ccccc1</smiles>

N-iso-Butylaniline ${ }^{3}$ (Table 2, entry 6 and Table 4, entry 2)<smiles>CCCNc1ccccc1</smiles>

$\boldsymbol{N}$-Pentylaniline (Table 2, entry 7 and Table 4, entry 3, commercially available)<smiles>CC(C)CNc1ccccc1</smiles>

$N$-(3-Methylbutyl)aniline (Table 2, entry 8 and Table 4, entry 4) Colorless oil. ${ }^{1} \mathrm{H}$ NMR $\left(\mathrm{CDCl}_{3}\right): \delta 7.17(\mathrm{t}, J=7.6 \mathrm{~Hz}, 2 \mathrm{H}), 6.69(\mathrm{t}, J=7.6 \mathrm{~Hz}, 1 \mathrm{H}), 6.61(\mathrm{~d}, J=7.6 \mathrm{~Hz}, 2 \mathrm{H})$, 3.54 (brs, NH), 3.12 (t, $J=7.3 \mathrm{~Hz}, 2 \mathrm{H}), 1.74-1.69$ (m, 1H), 1.52 (q, J=7.3 Hz, 2H), 0.95 (d, $J=6.4 \mathrm{~Hz}, 6 \mathrm{H}) .{ }^{13} \mathrm{C} \mathrm{NMR}\left(\mathrm{CDCl}_{3}\right): \delta .148 .5,129.2,117.1,112.7,42.1,38.6,26.0$, 22.6. MS (EI) $m / z 163\left(\mathrm{M}^{+}, 20 \%\right), 106$ (100), 77 (14). HRMS (EI) Calcd for $\mathrm{C}_{11} \mathrm{H}_{17} \mathrm{~N}$ $\left(\mathrm{M}^{+}\right)$163.1354. Found 163.1361. 
$\mathrm{NHCH}_{2} \mathrm{C}\left(\mathrm{CH}_{3}\right)_{3}$

$\mathrm{N}$-(2,2-Dimethylpropyl)aniline (Table 2, entry 10) Colorless oil. ${ }^{1} \mathrm{H} \mathrm{NMR}\left(\mathrm{CDCl}_{3}\right): \delta$ 7.16 (t, $J=7.4 \mathrm{~Hz}, 2 \mathrm{H}), 6.67$ (t, $J=7.4 \mathrm{~Hz}, 1 \mathrm{H}), 6.62$ (d, $J=7.4 \mathrm{~Hz}, 2 \mathrm{H}), 3.62$ (brs, NH), $2.89(\mathrm{~s}, 2 \mathrm{H}), 0.99(\mathrm{~s}, 9 \mathrm{H}) .{ }^{13} \mathrm{C} \mathrm{NMR}\left(\mathrm{CDCl}_{3}\right): \delta .149 .1,129.2,116.9,112.6,55.8,31.8$, 22.7. MS (EI) $m / z 163\left(\mathrm{M}^{+}, 15 \%\right), 106$ (100), 77 (13). HRMS (EI) Calcd for $\mathrm{C}_{11} \mathrm{H}_{17} \mathrm{~N}$ $\left(\mathrm{M}^{+}\right)$163.1365. Found 163.1361.

NH(CH

$\boldsymbol{N}$-Dodecylaniline (Table 2, entry 12 and Table 4, entry 5, commercially available)<smiles>c1ccc(NCC2CCCCC2)cc1</smiles>

$N$-(Cyclohexylmethyl)aniline ${ }^{4}$ (Table 2, entry 13)<smiles>OCCNc1ccccc1</smiles>

$N$-(3-Hydroxypropyl)aniline ${ }^{5}$ (Table 2 , entry 14)<smiles>N#CCNc1ccccc1</smiles>

$\boldsymbol{N}$-(3-Cyanopropyl)aniline (Table 2, entry 15) Colorless oil. ${ }^{1} \mathrm{H} \mathrm{NMR}\left(\mathrm{CDCl}_{3}\right): \delta 7.19$ (t, $J=7.3 \mathrm{~Hz}, 2 \mathrm{H}), 6.74$ (t, $J=7.3 \mathrm{~Hz}, 1 \mathrm{H}), 6.62$ (d, $J=7.3 \mathrm{~Hz}, 2 \mathrm{H}), 3.69$ (brs, NH), 3.32 (t, $J=6.7 \mathrm{~Hz}, 2 \mathrm{H}), 2.48(\mathrm{t}, J=6.7 \mathrm{~Hz}, 2 \mathrm{H}), 2.00-1.94(\mathrm{~m}, 2 \mathrm{H}) .{ }^{13} \mathrm{C} \mathrm{NMR}\left(\mathrm{CDCl}_{3}\right): \delta 147.5$, 129.4, 119.3, 118.0, 112.8, 42.3, 25.3, 14.8. MS (EI) $m / z 160\left(\mathrm{M}^{+}, 25 \%\right), 106$ (100), 77 (15). HRMS (EI) Calcd for $\mathrm{C}_{10} \mathrm{H}_{12} \mathrm{~N}_{2}\left(\mathrm{M}^{+}\right)$160.0992. Found 160.1001. 


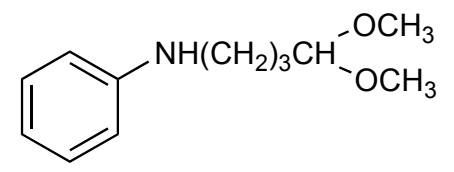

$N$-(4,4-Dimethoxybutyl)aniline (Table 2, entry 16) The procedure afforded the mixture $\left(680.0 \mathrm{mg}\right.$ ) of the title compound and nitrile. The structure was determined by ${ }^{1} \mathrm{H}$ NMR and HRMS data of the crude mixture.

$\mathrm{NH}^{\mathrm{NH}\left(\mathrm{CH}_{2}\right)_{2} \mathrm{Ph}}$

$N$-(2-Phenylethyl)aniline ${ }^{6}$ (Table 2, entry 15)<smiles>CCNc1cc(OC)c(OC)c(OC)c1</smiles>

$N$-Ethyl-3,4,5-trimethoxyaniline (Table 3, entry 1) Dark blue oil. ${ }^{1} \mathrm{H}$ NMR $\left(\mathrm{CDCl}_{3}\right)$ : $\delta$ $5.85(\mathrm{~s}, 2 \mathrm{H}), 3.83(\mathrm{~s}, 6 \mathrm{H}), 3.76(\mathrm{~s}, 3 \mathrm{H}), 3.13(\mathrm{t}, J=7.1 \mathrm{~Hz}, 2 \mathrm{H}), 1.26(\mathrm{t}, J=7.1 \mathrm{~Hz}, 3 \mathrm{H})$. ${ }^{13} \mathrm{C} \mathrm{NMR}\left(\mathrm{CDCl}_{3}\right): \delta 153.9,145.2,130.0,90.3,61.1,55.9,38.9,14.8$. MS (EI) $\mathrm{m} / z 211$ $\left(\mathrm{M}^{+}, 40 \%\right), 196$ (100). HRMS (EI) Calcd for $\mathrm{C}_{11} \mathrm{H}_{17} \mathrm{NO}_{3}\left(\mathrm{M}^{+}\right)$211.1196. Found 211.1209.<smiles>CCNc1ccc(NC(C)=O)cc1</smiles>

4-Ethylaminoacetanilide (Table 3, entry 2) White solid. Mp $123-124{ }^{\circ} \mathrm{C} .{ }^{1} \mathrm{H}$ NMR $\left(\mathrm{CDCl}_{3}\right): \delta 7.25(\mathrm{~d}, J=8.5 \mathrm{~Hz}, 2 \mathrm{H}), 6.55$ (d, $\left.J=8.5 \mathrm{~Hz}, 2 \mathrm{H}\right), 3.50$ (brs, NH), 3.12 (q, $J=7.1 \mathrm{~Hz}, 2 \mathrm{H}), 2.11(\mathrm{~s}, 3 \mathrm{H}), 1.25(\mathrm{t}, J=7.1 \mathrm{~Hz}, 3 \mathrm{H}) .{ }^{13} \mathrm{C} \mathrm{NMR}\left(\mathrm{CDCl}_{3}\right): \delta$ 168.3, 145.6, 128.1, 122.4, 112.8, 38.7, 24.1, 14.8. MS (EI) m/z 167 (M+, 100\%), 163 (40), 121 (65). Anal. Calcd for $\mathrm{C}_{10} \mathrm{H}_{14} \mathrm{~N}_{2} \mathrm{O}$ : C, 67.39; H, 7.92; N, 15.72. Found C, 67.39; H, 8.08; N, 15.67.

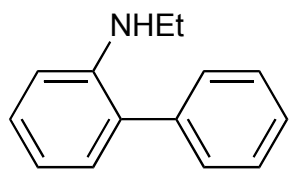

2-Ethylaminobiphenyl ${ }^{7}$ (Table 3 , entry 3) 
NHEt

$\boldsymbol{N}$-Ethyl-4-fluoroaniline (Table 3, entry 4) Brown oil. ${ }^{1} \mathrm{H}$ NMR $\left(\mathrm{CDCl}_{3}\right): \delta$ 6.93-6.86 (m, 2H), 6.55-6.52 (m, 2H), 3.41 (brs, NH), 3.11 (q $J=7.1 \mathrm{~Hz}, 2 \mathrm{H}), 1.25$ (t, $J=7.1 \mathrm{~Hz}$, $3 \mathrm{H}) .{ }^{13} \mathrm{C}$ NMR $\left(\mathrm{CDCl}_{3}\right): \delta 156.9,154.6,144.8,115.7,155.5,113.5,113.5,39.1,14.9$. MS (EI) $m / z 139\left(\mathrm{M}^{+}, 38 \%\right), 124$ (100). HRMS (EI) Calcd for $\mathrm{C}_{8} \mathrm{H}_{10} \mathrm{FN}\left(\mathrm{M}^{+}\right)$139.0797. Found 139.0792.<smiles>CCNc1ccc(C(C)=O)cc1</smiles>

4-Ethylaminobenzoic acid (Table 3, entry 5) White solid. Mp 179-180 ${ }^{\circ} \mathrm{C} .{ }^{1} \mathrm{H}$ NMR $\left(\mathrm{CDCl}_{3}\right): \delta 7.92(\mathrm{~d}, J=8.8 \mathrm{~Hz}, 2 \mathrm{H}), 6.55$ (d, $\left.J=8.8 \mathrm{~Hz}, 2 \mathrm{H}\right), 3.23(\mathrm{q}, J=7.1 \mathrm{~Hz}, 2 \mathrm{H}), 1.29$ $(\mathrm{t}, J=7.1 \mathrm{~Hz}, 3 \mathrm{H}) .{ }^{13} \mathrm{C} \mathrm{NMR}\left(\mathrm{CDCl}_{3}\right): \delta 171.7,152.6,132.3,117.1,111.3,37.9,14.6$. MS (EI) $m / z 165\left(\mathrm{M}^{+}, 45 \%\right), 150$ (100). Anal. Calcd for $\mathrm{C}_{9} \mathrm{H}_{11} \mathrm{NO}_{2}: \mathrm{C}, 65.44 ; \mathrm{H}, 6.71 ; \mathrm{N}$, 8.48. Found C, 65.22; H, 6.67; N, 8.32.<smiles>CCNc1ccccc1C(=O)O</smiles>

$N$-Ethylanthranilic acid (Table 3, entry 6) Light brown solid. Mp 156-157 ${ }^{\circ}$. ${ }^{1} \mathrm{H}$ NMR $\left(\mathrm{CDCl}_{3}\right): \delta 7.96(\mathrm{~d}, J=8.0 \mathrm{~Hz}, 1 \mathrm{H}), 7.39$ (t, $\left.J=8.0 \mathrm{~Hz}, 1 \mathrm{H}\right), 6.69$ (d, $\left.J=8.0 \mathrm{~Hz}, 1 \mathrm{H}\right), 6.60$ (t, $J=8.0 \mathrm{~Hz}, 1 \mathrm{H}), 3.26(\mathrm{q}, J=7.1 \mathrm{~Hz}, 2 \mathrm{H}), 1.32(\mathrm{t}, J=7.1 \mathrm{~Hz}, 3 \mathrm{H}) .{ }^{13} \mathrm{C}$ NMR $\left(\mathrm{CDCl}_{3}\right)$ : $\delta 173.0,151.8,135.5,132.6,114.5,111.3,108.5,37.4,14.5$. MS (EI) $m / z 165\left(\mathrm{M}^{+}\right.$, 50\%), 132 (100), 150 (25), 77 (19), 57 (15). Anal. Calcd for $\mathrm{C}_{9} \mathrm{H}_{11} \mathrm{NO}_{2}$ : C, 65.44; $\mathrm{H}$, $6.71 ; \mathrm{N}, 8.48$. Found C, 65.58; H, 6.65; N, 8.26.<smiles>CCNc1ccc(C(F)(F)F)cc1</smiles>

$\mathrm{N}$-Ethyl-4-trifluoromethylaniline (Table 3, entry 8) Colorless oil. ${ }^{1} \mathrm{H}$ NMR $\left(\mathrm{CDCl}_{3}\right)$ : $\delta$ 7.39 (d, $J=8.5 \mathrm{~Hz}, 2 \mathrm{H}), 6.58$ (t, $J=8.5 \mathrm{~Hz}, 2 \mathrm{H}), 3.89$ (brs, NH), 3.22-3.15 (m, 2H), 1.27 $(\mathrm{t}, J=7.3 \mathrm{~Hz}, 3 \mathrm{H}) .{ }^{13} \mathrm{C} \mathrm{NMR}\left(\mathrm{CDCl}_{3}\right): \delta 150.8,126.7,126.6,126.6,111.7,38.1,14.6$. MS (EI) $m / z 189\left(\mathrm{M}^{+}, 30 \%\right), 174$ (100). HRMS (EI) Calcd for $\mathrm{C}_{9} \mathrm{H}_{10} \mathrm{~F}_{3} \mathrm{~N}\left(\mathrm{M}^{+}\right)$189.0765. 
Found 189.0771.

NHEt

$\boldsymbol{N}$-Ethyl- $\beta$-naphthylamine (Table 3 , entry 9$)$ Brown oil. ${ }^{1} \mathrm{H} \mathrm{NMR}\left(\mathrm{CDCl}_{3}\right): \delta 7.65(\mathrm{~d}$, $J=8.0 \mathrm{~Hz}, 1 \mathrm{H}), 7.61(\mathrm{~d}, J=8.0 \mathrm{~Hz}, 2 \mathrm{H}), 7.35$ (t, $J=8.0 \mathrm{~Hz}, 1 \mathrm{H}), 7.18$ (t, $J=8.0 \mathrm{~Hz}, 1 \mathrm{H})$, $6.86(\mathrm{~d}, J=8.0 \mathrm{~Hz}, 1 \mathrm{H}), 6.80$ (s, 1H), 3.71 (brs, NH), 3.26 (q, J=7.2 Hz, 2H), 1.32 (t, $J=7.2 \mathrm{~Hz}, 3 \mathrm{H}) .{ }^{13} \mathrm{C} \mathrm{NMR}\left(\mathrm{CDCl}_{3}\right): \delta 146.6,135.3,128.9,127.6,127.5,126.3,125.9$, 121.8, 118.0, 104.3, 38.5, 14.8. MS (EI) m/z $171\left(\mathrm{M}^{+}, 75 \%\right), 156$ (100), 127 (40). HRMS (EI) Calcd for $\mathrm{C}_{12} \mathrm{H}_{13} \mathrm{~N}\left(\mathrm{M}^{+}\right)$171.1048. Found 171.1038.

$\overbrace{N}^{N H E t}$

$N$-Ethyl-3-aminopyridine ${ }^{8}$ (Table 3 , entry 10)

\section{$\mathrm{CH}_{3}\left(\mathrm{CH}_{2}\right)_{9} \mathrm{NEt}_{2}$}

$\boldsymbol{N}, \boldsymbol{N}$-Diethyldecylamine (Table 5, entry 1) Colorless oil. ${ }^{1} \mathrm{H} \mathrm{NMR}\left(\mathrm{CDCl}_{3}\right): \delta 2.51(\mathrm{t}$, $J=7.2 \mathrm{~Hz}, 4 \mathrm{H}), 2.40$ (t, $J=7.8 \mathrm{~Hz}, 2 \mathrm{H}), 1.47-1.42(\mathrm{~m}, 2 \mathrm{H}), 1.38-1.20$ (m, 14H), 1.02 (t, $J=7.2 \mathrm{~Hz}, 6 \mathrm{H}), 0.88(\mathrm{t}, J=6.8 \mathrm{~Hz}, 3 \mathrm{H}) .{ }^{13} \mathrm{C} \mathrm{NMR}\left(\mathrm{CDCl}_{3}\right): \delta 53.1,46.9,31.9,29.6,29.6$, 29.3, 27.8, 27.0, 22.7, 14.1, 11.7. MS (FAB, Gly) $m / z 214\left(\mathrm{M}^{+}+\mathrm{H}, 17 \%\right)$. HRMS (FAB, Gly) Calcd for $\mathrm{C}_{14} \mathrm{H}_{32} \mathrm{~N}\left(\mathrm{M}^{+}+\mathrm{H}\right) 214.2535$. Found 214.2530.

\section{$\mathrm{CH}_{3}\left(\mathrm{CH}_{2}\right)_{9} \mathrm{NBu}_{2}$}

$\boldsymbol{N}, \boldsymbol{N}$-Dibutyldecylamine (Table 5, entry 2 ) Colorless oil. ${ }^{1} \mathrm{H} \mathrm{NMR}\left(\mathrm{CDCl}_{3}\right): \delta 2.40-2.36$ $(\mathrm{m}, 6 \mathrm{H}), 1.44-1.37(\mathrm{~m}, 6 \mathrm{H}), 1.33-126(\mathrm{~m}, 18 \mathrm{H}), 0.93-0.86(\mathrm{~m}, 9 \mathrm{H}) .{ }^{13} \mathrm{C} \mathrm{NMR}\left(\mathrm{CDCl}_{3}\right)$ : $\delta 54.3,54.0,31.9,29.6,29.2,27.7,27.0,22.6,20.8,14.1$. MS (FAB, NBA) $m / z 270$ $\left(\mathrm{M}^{+}+\mathrm{H}, 52 \%\right)$. HRMS (EI) Calcd for $\mathrm{C}_{18} \mathrm{H}_{40} \mathrm{~N}\left(\mathrm{M}^{+}+\mathrm{H}\right) 270.3161$. Found 270.3158.

$\mathrm{CH}_{3}\left(\mathrm{CH}_{2}\right)_{9} \mathrm{~N}-\left(\mathrm{CH}_{2}\right)_{4} \mathrm{CH}_{3} \mathrm{CH}_{3}$

$\boldsymbol{N}, \boldsymbol{N}$-Dipentyldecylamine (Table 5, entry 3) Colorless oil. ${ }^{1} \mathrm{H}$ NMR $\left(\mathrm{CDCl}_{3}\right): \delta 2.37(\mathrm{t}$, $J=7.6 \mathrm{~Hz}, 6 \mathrm{H}), 1.46-1.20(\mathrm{~m}, 14 \mathrm{H}), 0.89-0.86(\mathrm{~m}, 9 \mathrm{H}) .{ }^{13} \mathrm{C} \mathrm{NMR}\left(\mathrm{CDCl}_{3}\right): \delta 54.1,31.9$, 29.8, 29.6, 29.3 , 27.6, 26.9, 22.6, 14.1. MS (FAB, Gly) m/z $298\left(\mathrm{M}^{+}+\mathrm{H}, 95 \%\right)$. HRMS (FAB, Gly) Calcd for $\mathrm{C}_{20} \mathrm{H}_{44} \mathrm{~N}\left(\mathrm{M}^{+}+\mathrm{H}\right)$ 298.3468. Found 298.3474. 


\section{$\mathrm{CH}_{3}\left(\mathrm{CH}_{2}\right)_{9} \mathrm{NHEt}$}

$\boldsymbol{N}$-Ethyldecylamine (Table 5, entry 4) Colorless oil. ${ }^{1} \mathrm{H}$ NMR $\left(\mathrm{CDCl}_{3}\right): \delta 2.64(\mathrm{q}, J=7.2$ $\mathrm{Hz}, 2 \mathrm{H}), 2.59$ (t, $J=7.1 \mathrm{~Hz}, 2 \mathrm{H}), 1.48-1.46(\mathrm{~m}, 2 \mathrm{H}), 1.28-1.26(\mathrm{~m}, 14 \mathrm{H}), 1.11(\mathrm{t}, J=7.2$ $\mathrm{Hz}, 3 \mathrm{H}), 0.88(\mathrm{t}, J=7.1 \mathrm{~Hz}, 3 \mathrm{H}) .{ }^{13} \mathrm{C} \mathrm{NMR}\left(\mathrm{CDCl}_{3}\right): \delta 50.0,44.2,31.9,30.3,29.6,29.3$, 27.4, 22.6, 15.4, 14.1. MS (FAB, NBA) $m / z 186\left(\mathrm{M}^{+}+\mathrm{H}, 20 \%\right)$. HRMS (FAB, NBA) Calcd for $\mathrm{C}_{12} \mathrm{H}_{27} \mathrm{~N}\left(\mathrm{M}^{+}+\mathrm{H}\right)$ 186.2228. Found 186.2222.

\section{$\mathrm{CH}_{3}\left(\mathrm{CH}_{2}\right)_{9} \mathrm{NHBu}$}

$\mathrm{N}$-Butyldecylamine (Table 5, entry 5) Colorless oil. ${ }^{1} \mathrm{H} \mathrm{NMR}\left(\mathrm{CDCl}_{3}\right): \delta$ 2.62-2.58 (m, 4H), 1.52-1.44 (m, 4H), 1.39-1.26 (m, 16H), 0.92 (t, $J=7.3 \mathrm{~Hz}, 3 \mathrm{H}), 0.88(\mathrm{t}, J=7.6 \mathrm{~Hz}$, $3 \mathrm{H}) .{ }^{13} \mathrm{C}$ NMR $\left(\mathrm{CDCl}_{3}\right): \delta 49.9,49.5,31.9,31.8,29.7,29.6,29.3,27.4,22.6,20.5,14.1$, 13.9. MS (FAB, NBA) $m / z 214\left(\mathrm{M}^{+}+\mathrm{H}, 15 \%\right)$. HRMS (FAB, NBA) Calcd for $\mathrm{C}_{14} \mathrm{H}_{32} \mathrm{~N}$ $\left(\mathrm{M}^{+}+\mathrm{H}\right)$ 214.2535. Found 214.2543.

\section{$\mathrm{CH}_{3}\left(\mathrm{CH}_{2}\right)_{9} \mathrm{NH}\left(\mathrm{CH}_{2}\right)_{4} \mathrm{CH}_{3}$}

$N$-Pentyldecylamine (Table 5, entry 6) Colorless oil. ${ }^{1} \mathrm{H}$ NMR $\left(\mathrm{CDCl}_{3}\right): \delta 2.58(\mathrm{t}, J=7.1$ $\mathrm{Hz}, 4 \mathrm{H}), 1.52-1.45$ (m, 4H), 1.36-1.26 (m, 18H), 0.90 (t, $J=7.1 \mathrm{~Hz}, 3 \mathrm{H}), 0.88$ (t, $J=7.1$ $\mathrm{Hz}, 3 \mathrm{H}) .{ }^{13} \mathrm{C} \mathrm{NMR}\left(\mathrm{CDCl}_{3}\right): \delta .50 .2,31.9,30.2,29.9,29.6,29.3,27.4,22.6,14.0 . \mathrm{MS}$ (EI) $m / z 227\left(\mathrm{M}^{+}, 10 \%\right), 170$ (100), 100 (100). HRMS (EI) Calcd for $\mathrm{C}_{15} \mathrm{H}_{33} \mathrm{~N}\left(\mathrm{M}^{+}\right)$ 227.2613. Found 227.2600. 


\section{References}

(1) Armarego, W. L. F.; Chai, C. L. L. Purification of Laboratory Chemicals, 5th ed.; Butterworth-Heinemann, Amsterdam, 2003; Although possible contaminants of commercial nitriles are the respective halides, alcohols (from hydrolysis), amines, amides, isonitriles, inorganic cyanides and so on, particular contaminants which influence the reported reaction is not clear yet.

(2) Holland, H. L.; Johnson, G. B. Tetrahedron Lett. 1979, 36, 3395-3396.

(3) Alonso, F.; Yus, M. Tetrahedron 1998, 54, 1921.

(4) Rische, T.; Eilbracht, P. Tetrahedron 1998, 54, 8441.

(5) Chini, M.; Crotti, P.; Favero, L.; Macchia, F. Tetrahedron Lett. 1994, 35, 761.

(6) Yang, J.-D.; Kim, M.-S.; Lee, M.; Baik, W.; Koo, S. Synthesis 2000, 789.

(7) Fadda, A. A.; Afsah, E. M. Indian Journal of Chemistry 1985, 24B, 970.

(8) Watanabe, Y.; Morisaki, Y.; Kondo, T.; Mitsuso, T. J. Org. Chem. 1996, 61, 4214. 